\title{
Effects of Astragalus injection and Salvia Miltiorrhiza injection on serum inflammatory markers in patients with stable coronary heart disease: a randomized controlled trial protocol
}

\author{
Zhihao Luo ${ }^{1 \dagger}$, Yuntao Liu ${ }^{1,2+}$, Zhen Zhao ${ }^{1}$, Xia Yan ${ }^{1,2}$, Dawei Wang ${ }^{1,3^{*}}$ and Qing Liu ${ }^{1,4^{*}}$ (I)
}

\begin{abstract}
Background: Coronary heart disease (CHD) is a clinical syndrome caused by coronary atherosclerosis (AS) or functional changes in coronary arteries. We have previously reported that astragaloside IV (in astragalus) and tanshinone IIA (in Salvia miltiorrhiza) improve myocardial ischemic injury.
\end{abstract}

Methods: This study will employ the randomized, blinded, prospective, single-center experiments approach. Briefly, a total of 160 eligible patients will be equally randomized into three treatment groups and placebo control groups. Patients will receive appropriate treatments every $24 \mathrm{~h}$ for seven days after enrollment and followed up for 28 days. The main evaluation indicators will be cell count, serum high-sensitivity C-reactive protein (hs-CRP) level, monocyte

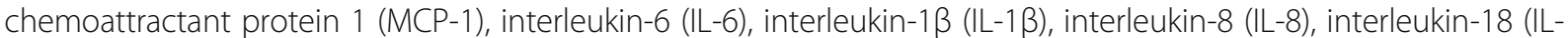
18), interleukin-10 (IL-10), tumor necrosis factor (TNF)-a, oxidized low density lipoprotein (OX-LDL), angina grade, and Traditional Chinese Medicine (TCM) symptom changes scale. Secondary indicators to be evaluated will include B-type natriuretic peptide (BNP) levels, troponin (CTn), muscle enzyme isoenzyme (CK-MB), heart-type fatty acid binding protein (H-FABP), liver and renal functions, as well as blood coagulation. Close monitoring of adverse events during the trial will also be conducted.

Discussion: This randomized controlled trial of Chinese herbal extracts for the treatment of coronary heart disease will generate results that are expected to provide valuable clinical evidence to inform future development of therapies towards management of CHD.

Trial registration: China Clinical Trial Registration Center, ChiCTR1900021590. Registered on 28 February 2019. Keywords: Stable coronary heart disease, Astragalus injection, Salvia miltiorrhiza injection, Randomized controlled trial

\footnotetext{
* Correspondence: 851757626@qq.com

${ }^{\dagger}$ Zhihao Luo and Yuntao Liu are co-authors

${ }^{1}$ Guangdong Provincial Hospital of Chinese Medicine, The Second Clinical

School of Medicine, Guangzhou University of Chinese Medicine, Guangzhou

and Zhuhai, China

Full list of author information is available at the end of the article
}

(c) The Author(s). 2020 Open Access This article is distributed under the terms of the Creative Commons Attribution 4.0 International License (http://creativecommons.org/licenses/by/4.0/), which permits unrestricted use, distribution, and reproduction in any medium, provided you give appropriate credit to the original author(s) and the source, provide a link to the Creative Commons license, and indicate if changes were made. The Creative Commons Public Domain Dedication waiver (http://creativecommons.org/publicdomain/zero/1.0/) applies to the data made available in this article, unless otherwise stated. 


\section{Background}

Coronary heart disease (CHD) is a clinical syndrome caused by coronary atherosclerosis (AS) or functional changes in the coronary arteries. This condition results in the narrowing of the vascular lumen and insufficient blood supply to the coronary arteries, thereby increasing the load on the heart and leads to acute and temporary ischemia as well as hypoxia of the myocardium. Coronary AS is a serious disease that endangers human health. In recent years, studies have found that AS is not only a simple lipid deposit, but also an inflammatory disease. Local or systemic inflammatory response plays an important role in the development of AS and its related complications [1]. Therefore, anti-inflammatory therapies have been proposed as the ideal means of treating AS. In fact, many studies have shown that statins, angiotensin-converting enzyme inhibitors (ACEIs), antiplatelet agents, calcium channel blockers, and other drugs have certain anti-inflammatory properties that can be exploited to manage the condition [2].

Inflammatory factors, endogenous peptides mainly produced by immune cells with strong biological effects, are known to be indicators of inflammatory responses. Specifically, they mediate a variety of immune responses, which are closely related to occurrence and development of coronary heart disease [3]. Studies have shown that serum high-sensitivity C-reactive protein (hs-CRP) is one of the markers of inflammation, helping to indicate an increased risk of vascular events, and aid prognosis and risk stratification. Consequently, this marker is a potential target for the treatment of AS and its complications [4]. Numerous reports have demonstrated a close relationship between level of hs-CRP, monocyte chemoattractant protein 1 (MCP-1), interleukin-6 (IL-6), interleukin-8 (IL-8), and tumor necrosis factor (TNF)- $\alpha$ with pathogenesis of coronary heart disease $[5,6]$. Since inflammatory factors are involved in the occurrence and development of $\mathrm{CHD}$, exploring the relationship between various inflammatory factors and coronary heart disease can provide a deeper understanding of the pathogenesis of this disease. In addition, quantification of various inflammatory factors can help to dynamically understand a patient's condition, while assessing the effects of treatment can guide clinical practice [7].

Coronary heart disease is categorized as "chest pain" and "heartache" in Traditional Chinese Medicine (TCM), which plays an important role in its diagnosis and treatment. In particular, the "Yiqihuoxue" is the most widely used method for managing the condition [8]. Among the components therein, astragalus and Salvia are the most commonly used compatibility drugs. In our previous studies, we focused on investigating the roles of TCM in CHD [9-12]. In particular, it is evident that astragalus membranaceus, the main component of astragalus membranaceus, and tanshinone IIA, the main component of Salvia miltiorrhiza, can effectively improve myocardial ischemic injury [13]. Functionally, the mechanism of action of the Salvia miltiorrhiza is related to inhibition of cell inflammatory reaction and apoptosis in myocardial ischemic injury [14].

Based on the above understanding, we hypothesize, from a clinical perspective, that individual astragalus and Salvia miltiorrhiza injections, or a combination of the two, have certain anti-inflammatory properties and could play a role in the treatment of CHD. We therefore aim to explore the effects of Salvia miltiorrhiza treatment on stable CHD and provide the basis for its clinical application.

\section{Methods/design}

Study design and settings

The present study will be set up using randomly blinded and single-center experiments. We will recruit 160 patients who meet the eligibility criteria at the Guangdong Provincial Hospital of Traditional Chinese Medicine. Subsequently, the participants will be divided into four equal groups: three groups with treatment (treatment group A will receive conventional drugs plus astragalus injection treatment, group B will receive conventional drugs plus Salvia miltiorrhiza injection, and group C will receive conventional drugs plus Salvia miltiorrhiza and astragalus injections) and the control group will receive conventional drugs plus $0.9 \% \mathrm{NaCl}$ injection. The study design is as illustrated in Fig. 1.

\section{Objectives}

This study intends to explore the effects of astragalus and Salvia miltiorrhiza injections as well as a synergistic treatment (of the two components) on stable CHD from the anti-inflammatory advantage and provide the basis for its clinical application.

\section{Research standard}

All patients enrolled in the study should meet the following diagnostic and inclusion criteria. In addition, the patients will sign an informed consent form (ICF) before the study.

\section{Diagnostic criteria}

The criteria for diagnosis of CHD will be as follows: (1) coronary angiography or coronary computed tomography (CT) examination confirmed at least one major branch lumen diameter stenosis of $>50 \%$, clinical with or without angina, heart failure, arrhythmia, sudden or death recovery; (2) clear evidence of ST-segment elevation or non-ST-segment elevation myocardial infarction; and (3) a history of percutaneous coronary intervention (PCI) and/or coronary artery bypass grafting (CABG). 


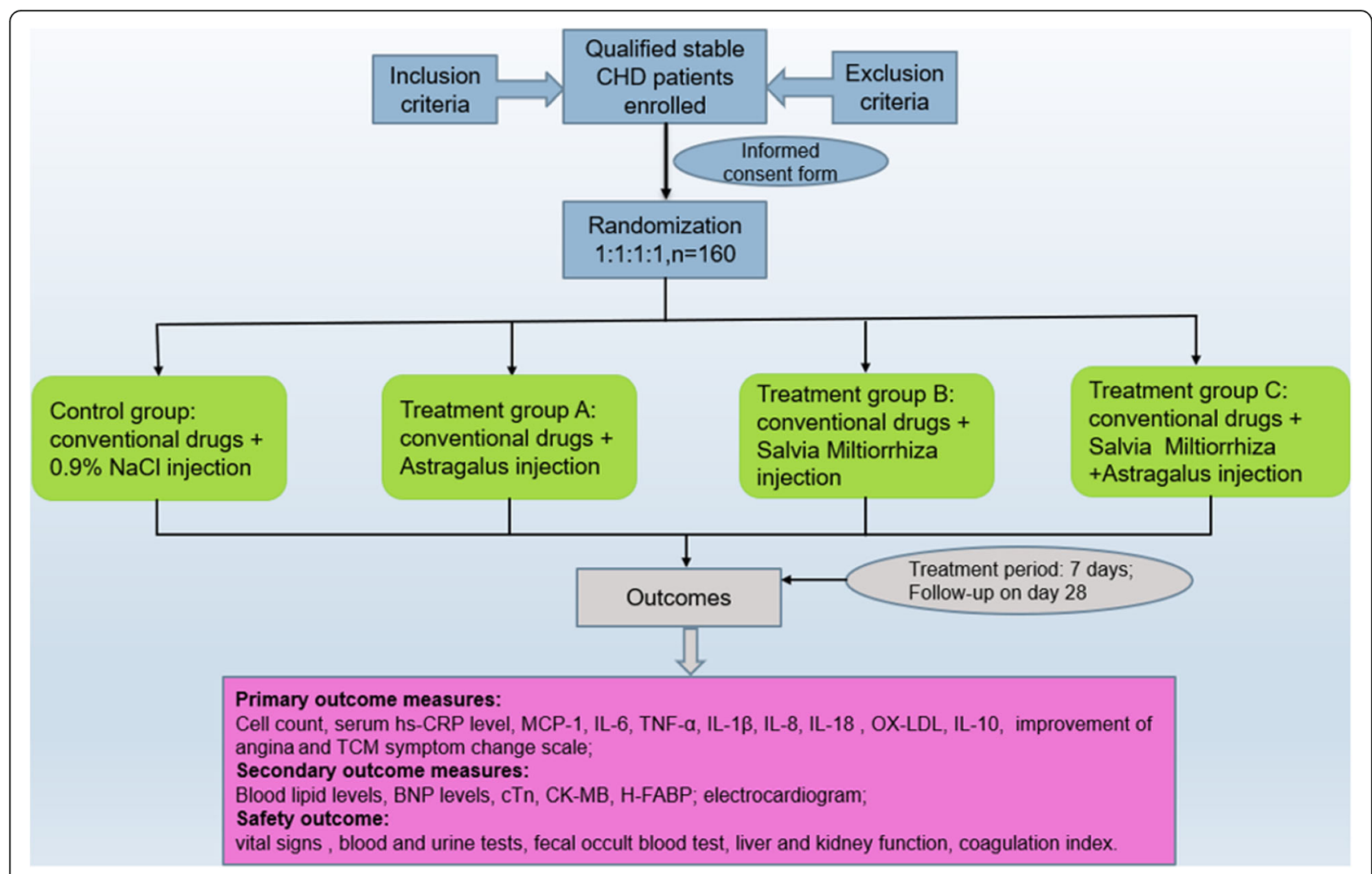

Fig. 1 Trial flow chart

Clinical diagnostic criteria for chronic stable angina will be as described [15]: chronic stable angina is a condition in which a patient's degree, frequency, nature, and predisposing factors of angina pectoris have not changed significantly within one month. The criteria will be as follows; (1) pain area: the typical part is the posterior sternum or left front chest, which can be radiated to the neck, pharynx, jaw, upper abdomen, shoulder and back, left arm, and left finger; (2) nature of pain: patients that often show symptoms of tightness, squeezing, pressure, burning, chest, chest tightness or suffocation, heavy feeling, subjective feelings vary widely, and some manifest as fatigue and shortness of breath; (3) duration: a paroxysmal episode lasting a few minutes, generally no more than $10 \mathrm{~min}$; and (4) predisposing factors and mitigation methods: patients whose seizures are related to labor or emotional excitement can be relieved after stopping to rest and sublingual nitroglycerin can quickly relieve symptoms within $2-5 \mathrm{~min}$.

\section{Inclusion criteria}

The inclusion criteria are as follows: (1) patients meet the stable coronary atherosclerotic heart disease (SCAD) diagnostic criteria; (2) their medical history is $>3$ months, frequency of angina pectoris is $\geq 3$ times in the past one week, and the severity of angina pectoris is grade I-II/I-III; (3) patient's age is $18-75$ years; (4) patients with SCAD treated with any combination of cardiac-related drugs for $>3$ months before enrollment, including atorvastatin, aspirin, angiotensin-II receptor blockers (ARBs), ACEIs, and beta-receptor blockers; and (5) patients signed the ICF.

\section{Exclusion criteria}

The exclusion criteria are as follows: (1) patients with acute coronary syndrome (ACS) who are asymptomatic or have stable symptoms after stabilization; (2) patients requiring revascularization, including myocardial infarction, left ventricular dysfunction, multivessel disease, and/or large-scale myocardial ischemia (ischemic area $>10 \%$ ), left main disease; (3) patients with chronic refractory angina pectoris; (4) patients with chronic kidney disease and severe heart, lung, liver, and other important organ dysfunction; serious primary diseases such as hematopoietic system and endocrine system, malignant tumor, gastrointestinal bleeding, gastric ulcer, and bleeding tendency are not expected complete the tester; (5) patients with severe and uncontrolled hypertension (systolic blood pressure $\geq 180 \mathrm{mmHg}$ or diastolic blood pressure $\geq 110$ $\mathrm{mmHg}$ ); (6) those with severe arrhythmia (ventricular dichotomy, ventricular tachycardia, ventricular fibrillation, third degree atrioventricular block, arrest, severe sinus 
bradycardia, sick sinus syndrome, re-entrant supraventricular tachycardia, arrhythmia causing hemodynamic changes); (7) patients who participate in other clinical trials or are taking other proprietary Chinese medicines within one month; (8) pregnancy, or preparation for pregnancy, and lactating women; and (9) people with mental, psychological, and other problems that cannot be matched with the completion of the research.

\section{Loss to follow-up}

Patients who are enrolling but have not completed clinical observations, including those withdrawing by themselves, losing their follow-up, poor compliance, or being withdrawn by a physician. If there are valid data in the case of shedding, the result of the last major efficacy index will be converted to the final result for statistical analysis.

\section{Suspension criteria}

The trial will be suspended in case of the following: (1) serious adverse reactions occurred during the testing; and (2) the trial finds that the clinical trial program has major errors, or the program is good, but serious deviations occur during implementation, and it is difficult to evaluate the efficacy of the drug.

\section{Sample size}

This study will use a parallel design of randomized controlled trials and hs-CRP as the main effect index. Conventional Western medicine treatment can reduce hsCRP by $32 \%$, according to clinical conditions, and it is estimated that Chinese medicine can reduce hs-CRP by $52 \%$. The set $\alpha=0.025, \beta=0.2$, superiority limit value $\delta=20 \%$, and the sample size of each group will be calculated as 30 cases $[16,17]$. Considering the loss of followup, 40 patients with stable coronary heart disease and 160 with four combinations will be selected.

\section{Randomization}

Random numbers will be generated using Statistics Analysis System (SAS) 9.2 software according to the principle of randomization. In order to implement random hiding, the random sequence will be kept by a trial manager. Using the remote random concealment method, it is determined that the basic information of qualified research individuals will be transferred by phone to the person who keeps the random numbers, from which the treatment allocation plan for each patient is obtained. Randomization of groups will be done according to a pre-made random scheme.

\section{Blinding}

Due to the special color problem of the TCM injection, we anticipate that it will be difficult to achieve doubleblinding during study. However, in order to avoid bias during the process, the following specific measures are proposed: (1) the researchers strictly adhere to the principle of randomization; (2) the doctors who participate in evaluation of the outcome are non-researchers, to ensure that evaluation of the efficacy of the test is blind; and (3) blood tests, data analysis, and statisticians to be covered up until the final analysis is completed. They only obtained the case report from (CRF) form and related data for analysis and did not understand the patient and drug use.

\section{Intervention}

Trial treatment methods

According to the principle of randomization, the group to be screened will be divided into four groups. The three groups of treatment are as follows: Group A, which receives conventional drug treatment plus astragalus injection treatment (size $20 \mathrm{~mL} /$ support, packaging 10/container), Group B, which will receive conventional medicine plus Salvia miltiorrhiza injection (specification $20 \mathrm{~mL} /$ support, packaging 10/container) and Group C, which will receive conventional medicine plus an injection of Salvia miltiorrhiza combined with astragalus. Both Salvia Miltiorrhiza and Astragalus injections will be prepared with $250 \mathrm{~mL}$ of $5 \%$ glucose. The control group receives an injection containing $250 \mathrm{~mL}$ conventional drug plus $5 \%$ glucose. For diabetic patients, an injection of $250 \mathrm{~mL} 5 \%$ glucose will be changed to $250 \mathrm{~mL}$ $0.9 \% \mathrm{NaCl}$. All patients will receive an intravenous drip once a day for $60 \mathrm{~min}$ for a treatment period of seven days.

The conventional drugs used in the stable coronary heart disease test will be as described in the ESC Guidelines for the diagnosis and treatment of SCAD [18]. The standard is given as aspirin, clopidogrel, low molecular weight heparin, statins, nitrates, beta-blockers, ACEIs, and other drugs.

\section{Pharmaceutical and combination methods}

For allocation and combination of the drugs in this test, we will prepare Salvia miltiorrhiza injection and astragalus injection using $250 \mathrm{~mL}$ of $5 \%$ glucose. If the patient is diabetic, we will change $250 \mathrm{~mL}$ of $5 \%$ glucose injection into $250 \mathrm{~mL}$ of $0.9 \% \mathrm{NaCl}$. No other injections will be used simultaneously during intravenous infusion.

\section{Matters needing attention}

During the study, patients will receive the test drug within $24 \mathrm{~h}$ of enrollment. Conventional treatment of stable coronary heart disease (such as anti-poly drug, lipid-lowering drug, antihypertensive drug) will be carried out at the same time. Injections of astragalus and Salvia miltiorrhiza should not be used as a substitute.

\section{Content and data capture points}

The contents and key points of the data to be collected during the study include the screening period (one day), $24 \mathrm{~h}$ before recruitment, a treatment period of 1-7 days 
followed by daily recording of data, and follow-up on the 28th day after treatment.

\section{Outcome measures}

\section{Primary outcome measures}

Cell counts, serum detection by enzyme-linked immunosorbent assay, including serum hs-CRP level, MCP-1, IL6, TNF- $\alpha$, IL-1 $\beta$, IL-8, IL-18, OX-LDL, IL-10, improvement of angina [19], and TCM symptom change scale [20] are outlined in Tables 1 and 2.

\section{Secondary outcome measures}

The following measurements will also be taken; blood lipid levels; B-type natriuretic peptide (BNP); troponin (cTn); muscle enzyme isoenzyme (CK-MB); heart-type fatty acid binding protein (H-FABP); and electrocardiogram.

\section{Safety outcomes}

These are vital signs including blood, urine, and fecal routines, liver and renal functions as well as coagulation. These indicators will be closely monitored during this process.

\section{Observation records of adverse events}

Any adverse events that occur during the course of the trial, including laboratory abnormalities, must be carefully interrogated and tracked. We will therefore judge these events by their nature, severity, and drug relevance and strictly record their outcomes in the case report form.

\section{Research process record points}

The subject content and data at each time point, according to the patient's hospitalization period, will be recorded

Table 1 Angina pectoris subscale

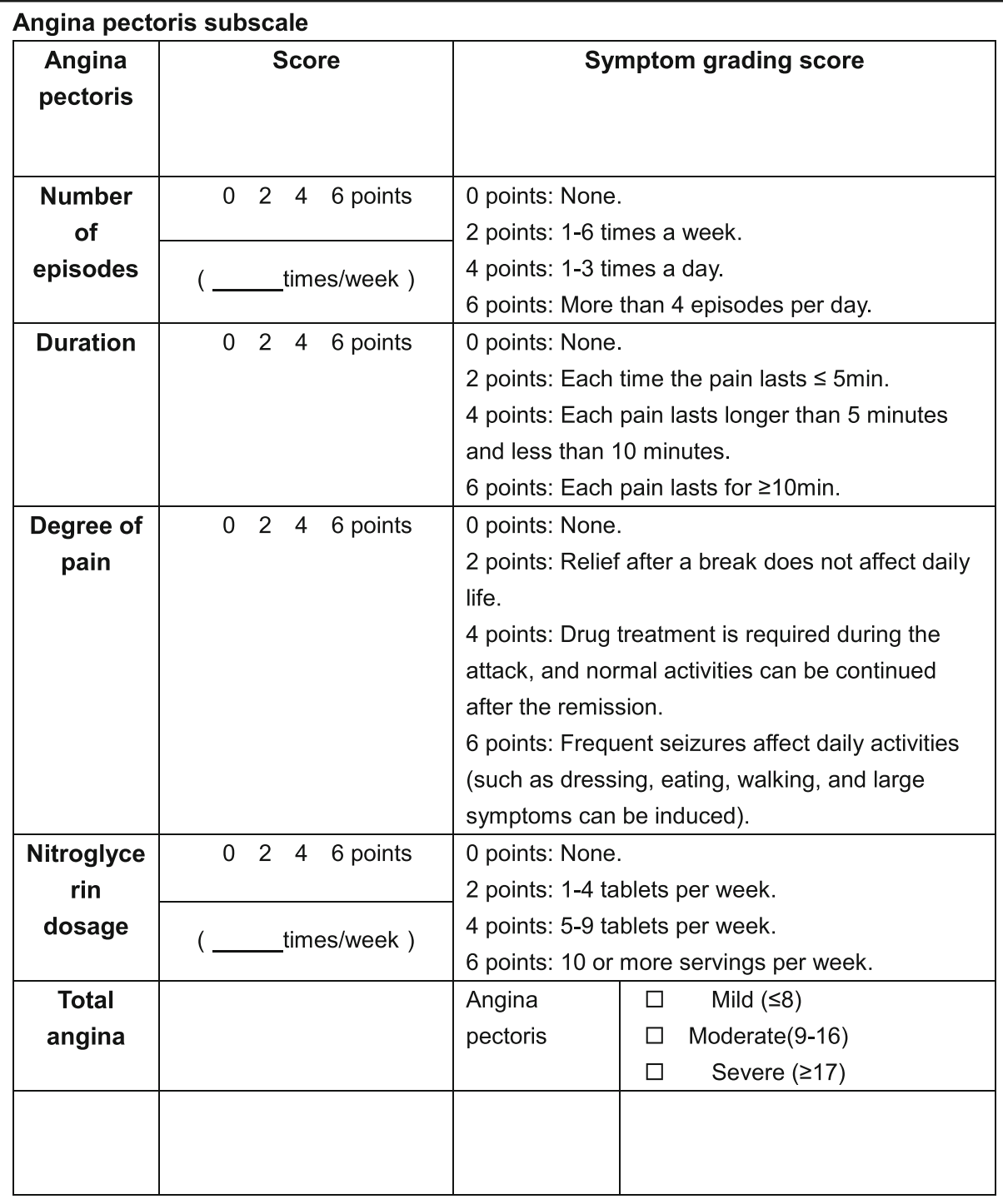


Table 2 Coronary heart disease Chinese medicine main symptom scale

\begin{tabular}{|c|c|}
\hline Symptom & Symptom grading score \\
\hline \multirow[t]{4}{*}{ Chest pain } & $\square 0$ points: None. \\
\hline & $\begin{array}{l}\square 3 \text { points: Remission after rest will not } \\
\text { affect daily life. }\end{array}$ \\
\hline & $\begin{array}{l}\square 6 \text { points: medication is required at the } \\
\text { time of onset, and normal life can be } \\
\text { continued after remission. }\end{array}$ \\
\hline & $\begin{array}{l}\square 9 \text { points: Frequent seizures affect daily } \\
\text { activities (such as dressing, eating, } \\
\text { walking, and large symptoms can be } \\
\text { induced). }\end{array}$ \\
\hline \multirow[t]{4}{*}{ Chest tightness } & $\square 0$ points: None. \\
\hline & $\begin{array}{l}\square 3 \text { points: I feel a chest tightness and } \\
\text { can relieve myself. }\end{array}$ \\
\hline & $\begin{array}{l}\square 6 \text { points: Chest tightness attacks are } \\
\text { more frequent, but do not affect } \\
\text { normal life and work. }\end{array}$ \\
\hline & $\begin{array}{l}\square 9 \text { points: Chest tightness continues } \\
\text { to be incomprehensible, affecting } \\
\text { life and work. }\end{array}$ \\
\hline \multirow[t]{4}{*}{ Shortness of breath } & $\square 0$ points: None. \\
\hline & $\square 2$ points: short after the event. \\
\hline & $\square 4$ points: A little move is short. \\
\hline & $\square 6$ points: I usually feel short of breath. \\
\hline \multirow[t]{4}{*}{ Weak } & $\square 0$ points: None. \\
\hline & $\square 2$ points: Severe activity is weak. \\
\hline & $\square 4$ points: Moderate activity is weak. \\
\hline & $\square 6$ points: Mild activity is weak. \\
\hline \multirow[t]{4}{*}{ Heart palpitations } & $\square 0$ points: None. \\
\hline & $\begin{array}{l}\square 1 \text { point: Occasionally, you can relieve } \\
\text { yourself. }\end{array}$ \\
\hline & $\begin{array}{l}\square 2 \text { points: Frequent attacks, but can } \\
\text { persist at work. }\end{array}$ \\
\hline & $\begin{array}{l}\square 3 \text { points: The heart is constantly } \\
\text { puzzled, affecting life and work. }\end{array}$ \\
\hline
\end{tabular}

as shown in Fig. 2. Specifically, the screening period $(0$ days) will be $24 \mathrm{~h}$ before recruitment followed by a daily data tracking and recording from day 1 to day 7 , and a follow-up on day 28 after treatment is administered.

\section{Statistical analysis}

Statistical analysis will be performed using SPSS 19.0 statistical software. Measured data will be described using means and standard deviation of the mean, while count data will be by the number of cases in percentages. First, a baseline analysis of the demographic characteristics in the four groups of selected cases will be conducted to investigate the balance and comparability across groups. Then, the effectiveness and safety indices will be compared across the four groups. The comparison of iso-quantitative data between the experimental and the control group will be performed using a student $t$-test of two-sample comparison for group design, while the comparison of the two classification indicators such as gender will be performed using the Cochran-MantelHaenszel $\chi^{2}$ test or Fisher's exact probability method. The missing supplementary data will be adjusted using imputation methods. To validate ineffectiveness among the groups, we will perform a rank sum test of the twosample comparison in the groups. All statistical tests will be two-sided with $p \leq 0.05$ to be used for statistical significance.

\section{Data management}

In the present study, the CRF will be filled out in time by the person in charge of the research. In addition, the patients will carefully fill in their relevant information and this will be regularly checked by clinical researchers to avoid missing data. The revisions provided by the clinical researcher will be implemented and corrected by the researcher in a timely manner. The researchers center will also confirm and sign the complete CRF form, in which no modifications are expected to be added after the trial. The entire research process will be conducted in a confidential manner, with none of the information disclosed to other parties. Only the lead researcher will have access to all data.

\section{Quality control}

Construction of research institutions and monitoring

During clinical research, multi-department construction and composition are required and each department should firmly cooperate to ensure the completion of quality research. In the current study, the main departments will include the following: (1) a competent department, composed of clinicians, pharmaceutical researchers, and quality supervision experts, which will be mainly responsible for the problems in clinical research, ensuring provision of solutions in a timely manner; (2) a Data Monitoring and Ethics Committee (DMEC): comprising experts in the field to monitor the overall conduct of the trial as well as relevant data and ensure that the rights of patients are adhered to; and (3) safety management: here, researchers from each center will be responsible for timely observations of the patient's vital signs and laboratory indicators, and generate timely adjustments and feedback.

\section{Supervision and inspection}

Effective supervision and inspection must be carried out throughout the clinical trial study. In this study, each researcher will conduct an examination once a week, including completion of a patient's CRF, verifying a complete informed consent, accurate patient's inclusion 


\begin{tabular}{|c|c|c|c|c|c|c|c|c|c|}
\hline \multirow[t]{2}{*}{ Visit } & \multirow{2}{*}{$\begin{array}{l}\text { Screening } \\
\text { V1 } \\
\end{array}$} & \multicolumn{7}{|c|}{ Intervention (7 days) } & \multirow{2}{*}{\begin{tabular}{|c|}
$\begin{array}{c}\text { Follow } \\
\text { up }\end{array}$ \\
vo
\end{tabular}} \\
\hline & & $\mathrm{V} 2$ & $\mathrm{~V}_{3}$ & $V_{4}$ & V5 & ve & $v_{7}$ & V8 & \\
\hline Unit: day & 0 & 1 & 2 & 3 & 4 & 5 & 6 & 7 & 28 \\
\hline Informed consent form & $\sqrt{ }$ & & & & & & & & \\
\hline Screening the subject & $\sqrt{ }$ & & & & & & & & \\
\hline Demographic information & $\sqrt{ }$ & & & & & & & & \\
\hline Inclusion/exclusion criteria & $\sqrt{ }$ & & & & & & & & \\
\hline Get random number & $\sqrt{ }$ & & & & & & & & \\
\hline History of SCAD & $\sqrt{ }$ & & & & & & & & \\
\hline Concomitant diseases & $\sqrt{ }$ & & & & & & & & \\
\hline Complication & $\sqrt{ }$ & & & & & & & & \\
\hline Vital signs & $\sqrt{ }$ & & & & & & & & \\
\hline Meet the SCAD diagnostic criteria & $\sqrt{ }$ & & & & & & & & \\
\hline the frequency of angina pectoris & $\sqrt{ }$ & & & & & & & & \\
\hline the severity of angina pectoris & $\sqrt{ }$ & & & & & & & & \\
\hline Age $18-75$ years old & $\sqrt{ }$ & & & & & & & & \\
\hline $\begin{array}{l}\text { Cell count, serum hs-CRP level, } \\
\text { MCP-1, IL-6, TNF- } \alpha \text {, IL-1 } \beta \text {, IL-8, } \\
\text { IL-18, OX-LDL, IL-10, }\end{array}$ & & $\sqrt{ }$ & & & & & & $\sqrt{ }$ & $\sqrt{ }$ \\
\hline improvement of angina & & $\sqrt{ }$ & & & & & & $\sqrt{ }$ & $\sqrt{ }$ \\
\hline TCM symptom change scale & & $\sqrt{ }$ & & & & & & $\sqrt{ }$ & $\sqrt{ }$ \\
\hline $\begin{array}{l}\text { Blood lipid levels, BNP levels, cTn, } \\
\text { CK-MB, H-FABP }\end{array}$ & & $\sqrt{ }$ & & & & & & $\sqrt{ }$ & $\sqrt{ }$ \\
\hline electrocardiogram & & $\sqrt{ }$ & & & & & & $\sqrt{ }$ & $\sqrt{ }$ \\
\hline Laboratory tests & & $\sqrt{ }$ & & & & & & $\sqrt{ }$ & $\sqrt{ }$ \\
\hline Safety outcomes & & $\sqrt{ }$ & & & & & & $\sqrt{ }$ & $\sqrt{ }$ \\
\hline Length of stay in hospitalization & $\sqrt{ }$ & $\sqrt{ }$ & $\sqrt{ }$ & $\sqrt{ }$ & $\sqrt{ }$ & $\sqrt{ }$ & $\sqrt{ }$ & $\sqrt{ }$ & $\sqrt{ }$ \\
\hline Cost of stay in hospitalization & $\sqrt{ }$ & $\sqrt{ }$ & $\sqrt{ }$ & $\sqrt{ }$ & $\sqrt{ }$ & $\sqrt{ }$ & $\sqrt{ }$ & $\sqrt{ }$ & $\sqrt{ }$ \\
\hline 28-day survival follow-up & & & & & & & & & $\sqrt{ }$ \\
\hline Efficacy and safety evaluation & & $\sqrt{ }$ & $\sqrt{ }$ & $\sqrt{ }$ & $\sqrt{ }$ & $\sqrt{ }$ & $\sqrt{ }$ & $\sqrt{ }$ & $\sqrt{ }$ \\
\hline
\end{tabular}

Fig. 2 Content and points of data capture

criteria, laboratory indicators and related scale collection, and preservation. During the test, patients with any adverse reactions and records of withdrawal will be identified. The researchers' center will ensure authenticity and integrity of all data.

\section{Ethical issues}

\section{Ethics statement}

In clinical trials, researchers will comply with the Helsinki Declaration and ethical principles. The investigator will fully respect the individuals' choices and will not force the individual to participate in the trial. The individual has the right to voluntarily participate, provide, and sign a written ICF, and may withdraw at any time. During recruitment, each participant will be assigned a random number to ensure blind implementation during the trial. In addition, researchers must protect the privacy and confidentiality of the individual.

\section{Ethical approval}

This study has been approved by the Ethics Committee of the Guangdong Provincial Hospital of Traditional Chinese Medicine on 1 February 2019.A clinical research plan and the relevant documents of the ICF are approved by the Ethics Committee of the Guangdong Provincial Hospital of Traditional Chinese Medicine. The approval registration number is BF2018-182-01 (Table 3).

\section{Informed consent form}

The ICF will be fully explained to the individual or guardian by the researcher. Those agreeing to participate will sign the ICF, and both the researcher and subject 
Table 3 Ethics committee names and approval registration number

\begin{tabular}{ll}
\hline Ethics committees' name & Approval registration number \\
\hline Ethics Committee of Guangdong Provincial Hospital of Traditional Chinese Medicine & BF2018-182-01 \\
\hline
\end{tabular}

The hospital has approved qualification for drug clinical trials from the China Food and Drug Administration (CFDA) and the investigator had a Good Clinical Practice (GCP) certificate

will keep the signed ICF individually. Project managers will be allowed access to the ICF kept by researchers for monitoring and inspection.

\section{Discussion}

CHD is a clinical syndrome caused by coronary AS or coronary functional changes. The condition results in the narrowing of the lumen in the blood vessels, insufficient blood supply to the coronary arteries, leading to cardiac load, and causing acute, transient ischemia as well as hypoxia of the myocardium [21]. AS is one of the main causes of coronary heart and cerebrovascular diseases, which contribute to a high global morbidity and mortality. Despite management options such as medical thrombolysis, and interventional or surgical bypass surgery, coronary artery recanalization directly results in myocardial ischemic injury. Myocardial ischemic injury has become a major clinical problem and has attracted wide research attention globally [22]. However, there is still no definitive and effective clinical method for prevention and treatment of this condition. It is, therefore, imperative to develop effective drugs for preventing and treating myocardial ischemic injury.

Recent studies have shown that chronic inflammation of the intima may play an important role in the pathogenesis of coronary atherosclerotic heart disease [16, 23]. There is a low degree of chronic inflammatory disease in AS. In fact, the vascular injury-response hypothesis suggests that endothelial dysfunction alters the anti-inflammatory properties of the endothelium. Inflammation, therefore, plays a major role in the development of atherothrombotic thrombosis and triggers cardiovascular events. For instance, since the 1990s, clinical research studies have linked cardiovascular events to chronic inflammation [24]. Furthermore, the role of inflammation in development of AS and sensitivity to predicting cardiovascular events has been identified. Current reports confirm that the inflammatory process is involved in the initiation of atherosclerotic progression and secondary lesions [25].

TCM has been vastly implicated in the prevention and treatment of CHD. In fact, since the development of integrated Chinese and Western medicine, coupled with the gradual deepening of research, TCM has acquired its own advantages in clinical practice enabling achievement of good efficacy. The basic pathogenesis of CHD is deficiency and blood stasis [22]. A treatment therapy for this condition has involved the use of Salvia miltiorrhiza injection, a TCM, whose main components are Salvia miltiorrhiza and antibalm. According to the relevant studies in modern medicine, Salvia miltiorrhiza can enhance cardiac function, improve the strength of myocardial contraction, increase blood supply to the myocardium, expand the blood vessels outside the heart, and generate very good anti-inflammatory effects [14]. In addition, Salvia miltiorrhiza injection has been found to promote blood circulation and remove blood stasis. Studies have further reported that static dripping of Salvia miltiorrhiza injection can significantly reduce inflammatory factors in patients with acute coronary syndrome [16], while in patients with acute myocardial infarction, its intravenous injection can reduce the level of hs-CRP and relieve chest tightness and pain [17]. Similarly, astragalus injection has been reported to increase myocardial contractility, with good protective effects on myocardial cells. Consequently, it has been found to effectively improve the cardiovascular function of patients. The drug can also strengthen the phagocytic function of macrophages, improve conversion rate of $\mathrm{T}$ cells, promote synthesis of interferon, and improve activity of interleukin. Furthermore, it affects immune regulation, antiviral replication, inhibits allergens, and bacteriostasis. Reports have shown that ulinastatin combined with astragalus injection generates good clinical effects on inflammatory reactions after PCI, which can reduce the expression levels of inflammatory factors in patients and reduce the myocardial injury caused by ischemia and reperfusion, which has important clinical application value [23].

In our previous studies, we found that the main components of astragalus, astragaloside IV, Salvia viltiorrhiza, and tanshinone IIA can effectively improve myocardial ischemic injury $[13,26]$. Functionally, the mechanism of action of these drugs targeted cellular inflammation in myocardial ischemic injury and inhibited death. However, from a clinical point of view, it remains unclear whether individual astragalus and Salvia miltiorrhiza injections, or a combination of the two, result in significantly different anti-inflammatory properties, during the treatment of CHD. This study will explore the effects of the astragalus injection, the Salvia miltiorrhiza injection, and their synergistic treatment on stable CHD from the anti-inflammatory advantage, and provide the basis and basis for its clinical application. 


\section{Trial status}

Participant recruitment is currently underway. The version number / date is $2.0 / 20190601$.

The start date of the recruitment of this project is 1 June 2019 and the approximate date of recruitment is 1 June 2020, for a period of one year.

\section{Abbreviations}

ACEls: Angiotensin-converting enzyme inhibitor; ACS: Acute coronary syndrome; ARBs: Angiotensin-II receptor blockers; AS: Atherosclerosis; ASA: Aspirin enteric capsules; BNP: B-type natriuretic peptide; CABG: Coronary artery bypass grafting; CHD: Coronary heart disease; CKMB: Muscle enzyme isoenzyme; CRA: Clinical research associate; CRF: Case report form; cTn: Troponin; GCP: Good Clinical Practice; H-FABP: Heart-type fatty acid binding protein; hs-CRP: High-sensitivity C-reactive protein; ICF: Informed consent form; IL-10: Interleukin-10; IL-18: Interleukin-18; IL1及: Interleukin-1ß; IL-6: Interleukin-6; IL-8: Interleukin-8; MCP-1: Monocyte chemoattractant protein 1; OX-LDL: Oxidized low density lipoprotein; PCl: Percutaneous coronary intervention; PI: Principal investigator; SAS: Statistics Analysis System; SCAD: Stable coronary atherosclerotic heart disease; SFDA: State Food and Drug Administration; TCM: Traditional Chinese Medicine; TNF-a: Tumor necrosis factor; $\beta$ RB: $\beta$ - receptor blocker

\section{Acknowledgements}

We are grateful to all the researchers who participated in this trial for their help and efforts. We also thank Guangdong Provincial Hospital of Traditional Chinese Medicine for their support in the treatment of facilities, recruitment, and treatment of patients.

\section{Authors' contributions}

DW and $\mathrm{QL}$ designed the study. ZL, YL, and $X Y$ drafted the manuscript. QL finalized the manuscript. Critical comments and typesetting correction on the final version are made by ZZ. All authors read, revised, and approved the final manuscript.

\section{Funding}

This research is supported by National Natural Science Foundation of China (No. 81603460, to QL), Postdoctoral Science Foundation of China (No. 2015 M581533, to QL), Guangdong Provincial Bureau of Traditional Chinese Medicine (No. 20184015). The National Natural Science Foundation of China is supporting the overall idea of the entire subject research and data collection; The Postdoctoral Science Foundation of China is supporting provision of clinical research programs and technical support; while the Guangdong Provincial Bureau of Traditional Chinese Medicine is mainly responsible for financial support.

\section{Availability of data and materials}

Not applicable.

\section{Ethics approval and consent to participate}

This trial complies with the principles of Declaration of Helsinki and the regulations of quality management of clinical trials in China. The research activities in this trial have been approved by the Ethics Committee of Guangdong Provincial Hospital of Traditional Chinese Medicine. The committee's reference number is BF2018-182-01 (Table 3). All study participants will sign written ICFs before participating in the trial.

\section{Consent for publication}

Not applicable.

\section{Competing interests}

The authors declare that they have no competing interests.

\section{Author details}

${ }^{1}$ Guangdong Provincial Hospital of Chinese Medicine, The Second Clinical School of Medicine, Guangzhou University of Chinese Medicine, Guangzhou and Zhuhai, China. ${ }^{2}$ Guangdong Provincial Key Laboratory of Research on Emergency in Traditional Chinese Medicine, Emergency Department, Guangdong Provincial Hospital of Chinese Medicine, Guangzhou, China. ${ }^{3}$ Shunde Hospital of Guangzhou University of Chinese Medicine, Foshan,
China. ${ }^{4}$ Department of Immunology, School of Basic Medical Sciences, Fudan University, Shanghai, China.

Received: 9 August 2019 Accepted: 26 January 2020

Published online: 17 March 2020

\section{References}

1. Matusiak A, Chałubiński M, Broncel M, Rechciński T, Rudnicka K, Miszczyk E, et al. Putative consequences of exposure to Helicobacter pylori infection in patients with coronary heart disease in terms of humoral immune response and inflammation. Arch Med Sci. 2016:12(1):45-54.

2. Fan D, Yang Z, Yuan $Y$, Wu QQ, Xu M, Jin YG, et al. Sesamin prevents apoptosis and inflammation after experimental myocardial infarction by JNK and NF-kB pathways. Food Funct. 2017;8(8):2875-85

3. Chen LJ, Lim SH, Yeh YT, Lien SC, Chiu JJ. Roles of microRNAs in atherosclerosis and restenosis. J Biomed Sci. 2012;19(1):79.

4. Guo Y, Zhou W, Luo Y, Zhang D. Research progress on the relationship between high-sensitivity C-reactive protein and coronary heart disease. J Cardiovasc Rehabil Med. 2017;26(02):225-7 Article in Chinese.

5. Chen X. Changes of serum troponin $T$ and high-sensitivity C-reactive protein levels in patients with coronary heart disease before and after interventional therapy and their correlation with prognosis. Chin Lab Diagn. 2017;21(3):409-10 Article in Chinese.

6. Feng Q. Clinical significance of correlation between high-sensitivity Creactive protein, lipoprotein-associated phospholipase A2 and D-dimer and coronary heart disease patients. Int J Immunol. 2017:40(2):156-60 Article in Chinese.

7. Qiu Z, Lu D. Effect of continuous positive airway pressure on hypersensitive C-reactive protein in patients with coronary heart disease complicated with obstructive sleep apnea. J Pract Med. 2016:32(9):1440-2 Article in Chinese.

8. Wang J, Li J, Yao H. Study on the syndrome elements and syndrome combination of angina pectoris. Chin Med J. 2007:48(10):920-2 Article in Chinese.

9. Liu Q, Wang J, Liang Q, Wang D, Luo Y, Li J, et al. Sparstolonin B attenuates hypoxia-reoxygenation-induced cardiomyocyte inflammation. Exp Biol Med (Maywood). 2014;239(3):376-84.

10. Liu Q, Li J, Jubair S, Wang D, Luo Y, Fan D, et al. Sparstolonin B attenuates hypoxia-induced apoptosis, necrosis and inflammation in cultured rat left ventricular tissue slices. Cardiovasc Drugs Ther. 2014;28(5):433-9.

11. Liu Q, Li J, Liang Q, Wang D, Luo Y, Yu F, et al. Sparstolonin B suppresses rat vascular smooth muscle cell proliferation, migration, inflammatory response and lipid accumulation. Vascul Pharmacol. 2015;67:59-66.

12. Liu Q, Li J, Wang J, Li J, Janicki JS, Fan D. Effects and mechanisms of chinese herbal medicine in ameliorating myocardial ischemia-reperfusion injury. Evid Based Complement Alternat Med. 2013;2013:925625.

13. Wang D, Liu Y, Zhong G, Wang Y, Zhang T, Zhao Z, et al. Compatibility of Tanshinone IIA and Astragaloside IV in attenuating hypoxia-induced cardiomyocytes injury. J Ethnopharmacol. 2017;204:67-76.

14. Wang Y. Analysis of Clinical Pharmacology and Combination of Danshen injection and Huangqi injection. Chin Pract Med. 2016;11(09):192-3. Article in Chinese.

15. Cardiovascular Branch of Chinese Medical Association. Guidelines for the diagnosis and treatment of chronic stable angina pectoris. Chin J Cardiovasc Dis. 2007;35(3):195-206 Article in Chinese.

16. Li Q, Gan S, Huang H. Effect of sodium tanshinone IIA sulfonate on acute coronary syndrome and its effect on inflammatory factors. Mod J Integr Tradit Chin West Med. 2015:24(21):2284-6 Article in Chinese.

17. Ma J, Liang X. Effect of salvia miltiorrhiza injection on serum homocysteine, folic acid and C-reactive protein levels in ST-segment elevation acute myocardial death patients. Chin J Integr Tradit West Med. 2016;14(21):252830 Article in Chinese.

18. Montalescot G, Sechtem U, Achenbach S, Andreotti F, Arden C, Budaj A et al. 2013 ESC guidelines on the management of stable coronary artery disease: the task force on the management of stable coronary artery disease of the European Society of Cardiology. Eur Heart J. 2013;34(38):2949-3003.

19. LV $Y$, He $Y$, Yang J. Study on symptom scores of coronary heart disease with angina pectoris (qi deficiency and blood stasis syndrome). Chin Clini Pharmacol Ther. 2008;13(7):786-91 Article in Chinese.

20. Chinese Medicine Association and Cardiovascular Disease Club. Evaluation criteria for medical efficacy in angina pectoris. Chin J Exp Tradit Med Formulae. 2017;24(15):7-10 Article in Chinese. 
21. Ge J, Xu Y. Internal medicine. Beijing: People's Health Publishing House; 2013. p. 227-8. Article in Chinese.

22. Yang Y. A review of the current status and research progress of interventional therapy for coronary heart disease. Chin J Integr Tradit West Cardiol. 2016;4(22):11-2 Article in Chinese.

23. Lan Y, Chen X. Clinical study of ulinastatin combined with astragalus injection in the treatment of inflammatory reaction after $\mathrm{PCl}$ in patients with myocardial infarction. Drugs Clin. 2015;30(07):841-5 Article in Chinese.

24. Roberts WL. CDC/AHA workshop on markers of inflammation and cardiovascular disease: application to clinical and public health practice: laboratory tests available to assess inflammation--performance and standardization: a background paper. Circulation. 2004;110(25):e572-6.

25. Kaptoge S, Seshasai SR, Gao P, Freitag DF, Butterworth AS, Borglykke A, et al. Inflammatory cytokines and risk of coronary heart disease: new prospective study and updated meta-analysis. Eur Heart J. 2014;35(9):578-89.

26. Qu Y, Wang D, Yan X. Effect of astragaloside on autophagy in neonatal rat cardiomyocytes and inhibition of apoptosis induced by ischemia-induced cardiomyocytes. Tradit Chin Med Clin Pharmacol. 2014;25(2):169-72 Article in Chinese.

\section{Publisher's Note}

Springer Nature remains neutral with regard to jurisdictional claims in published maps and institutional affiliations.

Ready to submit your research? Choose BMC and benefit from:

- fast, convenient online submission

- thorough peer review by experienced researchers in your field

- rapid publication on acceptance

- support for research data, including large and complex data types

- gold Open Access which fosters wider collaboration and increased citations

- maximum visibility for your research: over $100 \mathrm{M}$ website views per year

At BMC, research is always in progress.

Learn more biomedcentral.com/submissions 